\section{Die Faktizität des Postfaktischen} Kommentar zu Robert Feustels „Substanz und Supplement. Mit Rechten reden, zu Rechten forschen?“
,Substanz und Supplement. Mit Rechten reden, zu Rechten forschen?"

Kommentare von: Regina Ammicht Quinn, Lee Hielscher, Annekatrin Kühn, Katrin Lehn, Nils Kumkar, Daniel Mullis, Andreas Nölke

Replik von: Robert Feustel
Der Zulauf rechter Bewegungen in den Jahren seit der Weltwirtschaftskrise ist eine bedrohliche Herausforderung für emanzipatorische Politik und damit auch für eine kritische Sozialforschung als „Moment einer auf neue gesellschaftliche Formen abzielenden Praxis“(Horkheimer 1937: 269). Diese Bewegungen stehen einem solchen Unterfangen nicht nur im Wege, sondern drohen, wo sie erfolgreich sind, auch deren bloße Möglichkeit zu unterminieren, spätestens wenn politischer Konflikt nur noch zwischen der Verteidigung des marktliberalen Status quo und seiner reaktionären Herausforderung erkenn- und artikulierbar ist.

Für die Entwicklung von Gegenstrategien ist es entscheidend, bessere Einblicke darin zu bekommen, warum bestimmte soziale Gruppen sich von diesen Bewegungen angesprochen fühlen. Feustels Ausgangsbeobachtung, dass es vor dem Hintergrund drängender gesellschaftlicher Problemlagen unumgänglich sei, dass kritische Sozialforscher_innen mit Rechten redeten (Feustel 2019: 137), ist deswegen meiner Meinung nach zutreffend. Genauso zutreffend ist die umfassende, wenn auch längst nicht erschöpfende Liste erkenntnistheoretischer, forschungsethischer und methodologischer Probleme, die sich bei einem solchen Unterfangen ergeben. Irritierend ist darum in meinen Augen Feustels (sicherlich als „Einladung zum Widerspruch“ gesuchte, zuspitzende) Bemerkung, dass es schlussendlich in Bezug auf die drängenden Fragen nur „bedingt“ (im Sinne von: eigentlich gar nicht) hilfreich sei (ebd.: 144), im Sinne der Forschung mit Rechten zu reden. Irritierend sind die Überlegungen, die zu diesem Schluss führen, auch deshalb, weil dabei eine lange und breite Tradition kritischer Sozialforschung, die sich den erwähnten Problemen gestellt und sie bearbeitet hat, einfach unter den Teppich gekehrt wird. Von Klassikern wie den Studien zum autoritären Charakter (Adorno 1973) und dem Frankfurter Gruppenexperiment der Nachkriegszeit (Pollock 1955) bis hin zu aktuellen Arbeiten wie den Leipziger Mitte-Studien (Decker/Kiess/Brähler 2015), Arlie Hochschilds (2017) Interviewstudie zu Unterstützer_innen der Rechten in den USA, die Feustel immerhin erwähnt, oder auch meinen eigenen Untersuchungen zur rechtspopulistischen Tea-Party-Bewegung und den Wähler_innen Donald 
Trumps (Kumkar 2017; 2018) hat die kritische Sozialforschung immer wieder mit Gewinn mit ,Rechten geredet'.

Einen guten Teil der Probleme der Forschung, die Feustel aufzählt, wie die oft besonders markante Kluft zwischen der von Rechtspopulist_innen bekundeten „Meinung“ (wie der rassistischen Abwertung von Geflüchteten) und ihren „eigentlichen Gründen“ im Sinne ihres aufzeigbaren sozialen Leidens (das mit den Geflüchteten oft gar nichts zu tun hat), oder die Persistenz „autoritärer Charakteranteile“ (Feustel 2019: 138) wären ohne die empirischen Ergebnisse dieser Forschung vermutlich nicht oder sehr viel weniger bekannt. Es ließe sich darüber hinausgehend sogar plausibel argumentieren, dass die deutschsprachige interpretative und rekonstruktive Sozialforschung ohne das Ringen mit den Problemen, die zum Beispiel die Erforschung der „Gegenwärtigkeit des ,Dritten Reichs“ in erzählten Lebensgeschichten“ (Rosenthal 1990) mit sich bringt, niemals ihre heutige Gestalt angenommen hätte. Es wird vor diesem Hintergrund nicht überraschen, dass ich anders als Feustel dafür plädiere, dass es durchaus sinnvoll, wenn nicht unumgänglich für eine kritische Sozialforschung ist, mit Rechten zu reden. Und zwar nicht trotz, sondern gerade wegen der aufgezählten Probleme, die ich viel eher im Sinne einer Fragestellung denn als Hindernisse begreifen würde.

Es ist dazu allerdings zunächst erforderlich, die etwas unglückliche alltagssprachliche Diktion der Frage, ob man mit Rechten reden solle, aufzubrechen und zumindest zu spezifizieren, was mit ,reden“ im Sinne der Forschung eigentlich sinnvoll gemeint sein kann. Gemeinhin verbirgt sich ja hinter der Forderung, man möge mit Rechten reden (oder eben gerade nicht), das Bild eines auf gegenseitiges Verständnis hin orientierten Gesprächs, bei dem nicht nur Einblicke in die Welt des_der jeweils anderen eröffnet werden, sondern auch auf dem Weg des Austauschs von Argumenten eine Annäherung der Positionen erreicht wird. In der sozialwissenschaftlichen empirischen Forschung ist dies jedoch keinesfalls die Regel und kann es meiner Meinung nach in vielen Forschungsbereichen auch gar nicht sein. Wenn ich also davon spreche, mit Rechten zu reden, dann meine ich zunächst einfach nur: Sich auf geeignetem Wege im Sinne der rekonstruktiven Sozialforschung einen Zugang zur gelebten Erfahrung und alltagspraktischen Auslegungspraxis der Befragten zu verschaffen, um deren innere Logik und gesellschaftliche Erzeugungsbedingungen zu verstehen.[1]

Denn auch wenn Feustel sicherlich Recht damit hat, eine besonders scharfe Kluft zwischen den „eigentlichen Gründen“ für die Unterstützung rechter Bewegungen und der artikulierten Meinung ihrer Anhänger_innen anzunehmen, ist das ja gerade eine Herausforderung für und kein Argument gegen die empirische Forschung - übrigens eine, die ganz generell und nicht nur im Fall der Rechten zu bedenken ist. Dass ,wir nie so ganz genau [wissen], wie das Verhältnis von sozialen Tatsachen oder Bedingungen und politischen Einstellungen beschaffen ist“" (Feustel 2019: 142), mag in dieser Allgemeinheit stimmen, geht aber wieder über vielversprechende Versuche, dieses Verhältnis theoretisch aufzuschlüsseln und damit für die empirische Forschung zu erschließen, hinweg (z. B. Bourdieu 1993; 2012). Die Aussage, dass, „,wer sich beständig im Kontrafaktischen bewegt“, „kaum von einer faktenbasierenden Wissenschaft gewinnbringend zu analysieren" sei (Feustel 2019: 143), wäre auf jeden Fall eine Kapitulationserklärung nicht 
nur vor dem Phänomen der rechten Bewegungen, sondern der empirischen Sozialforschung in einem umfassenderen Sinne. Denn nicht nur dass sie sich im Kontrafaktischen bewegen, ist ein Fakt, der sich belegen lässt, auch die Gründe dafür und für die spezifische Art, sich im Kontrafaktischen zu bewegen, sind als soziale Tatsachen wichtige Gegenstände der empirischen Untersuchung. Für die rekonstruktive Sozialforschung ganz allgemein gilt, dass „,[s]elbst ein vollkommen verderbter, bewusst gefälschter, nicht einmal mehr eine tatsächliche Pathologie gültig verkörpernder Text [...] immer noch eine Relation der Gültigkeit zu dieser Fälschungspraxis aufweisen“ wird (Oevermann 2005: 24) - selbst die Lüge ist, in anderen Worten, noch authentischer Ausdruck einer zu untersuchenden Praxis des Lügens.

Gerade um das Vermittlungsverhältnis von (makro-)sozialen Ursachen des momentanen Aufwinds der extremen Rechten und ihren Artikulationen zu verstehen, ist es deshalb unumgänglich, einen Einblick in die Lebenswelt der Betroffenen als Ensemble der Produktionsbedingungen ihrer ,Meinung zu erarbeiten. Der Verzicht auf die methodisch kontrollierte, empirische Grundierung der Überlegungen zu diesem komplexen Verhältnis umgeht diese Schwierigkeit nämlich nicht, sondern läuft erst recht Gefahr, eine besonders mustergültige Form des intellektuellen „Ethnozentrismus“ zu produzieren (Bourdieu 1979: 153). Das heißt nicht, dass man die Befragten einfach nach den „eigentlichen Gründen“ fragt, zu denen sie so direkt, da stimme ich Feustel wieder zu, vermutlich nicht besonders auskunftsfähig und -freudig sind,[2] und auch nicht, dass man sie nach ihrer „Meinung“ fragt, um dann die eigentlichen Gründe dahinter intuitiv oder theoretisch hineinzugeheimnissen. Die Aufgabe des "methodisch kontrollierten Fremdverstehens“ (Bohnsack 2010: 20) ist es vielmehr, möglichst valide Einblicke in die gelebte Erfahrung der Befragten zu gewinnen und dabei zu erkunden, warum gerade vor diesem Hintergrund die Anschlussfähigkeit bestimmter Klischees und Slogans gegeben ist und was deren Funktion für die eigene Handlungsund Deutungspraxis ausmacht. Dabei ergeben sich nicht nur interessante Einblicke in das Problem der Kluft zwischen dem alltäglichen Leben und dem Diskurs der Betroffenen und dem Stellenwert sogenannter „Fake-News“, sondern es wird überdies deutlich, dass es von zentraler Bedeutung ist, die sozialen Gruppen, die als Unterstützer_innen der Rechten auftreten, feiner zu differenzieren.

In meiner Forschung habe ich zum Beispiel narrative Interviews und Gruppendiskussionen mit Tea-Party-Anhänger_innen geführt, die es mir erlaubten, die lebensgeschichtlichen Hintergründe der Befragten zu rekonstruieren und zu beobachten, wie sie gemeinsam ihr Erleben und ihr Engagement in der Tea Party verhandelten.[3] Dabei stellte sich unter anderem heraus, dass die älteren Angehörigen des klassischen Kleinbürgertums, die die Kernklientel dieser Bewegung stellten, in all den verschiedenen Verschwörungstheorien und Untergangsszenarien immer wieder metaphorisch dieselbe Erfahrung wiederholten: Dass nämlich ihr lebenslanges Streben nach materiellem Wohlstand und sozialer Anerkennung über harte, selbstständige wirtschaftliche Tätigkeit nicht den versprochenen Gewinn brachte. Diese Krisenerfahrung formulierten sie aber in ihren Anekdoten jedes Mal dergestalt metaphorisch um, dass die darin drohende Angst vor dem eigenen Scheitern verdrängt und die Verantwortung zudem von den gesellschaftlich-ideologischen 
Instanzen von Marktwirtschaft und Leistungsethos, denen man weiterhin die Treue hielt, wegverschoben werden konnte. Die eigene biographische Krise konnten sie so als ,Enttäuschung ohne Desillusionierung verarbeiten. Die dabei formulierten Verschwörungstheorien mussten weder in sich konsistent noch miteinander kompatibel sein, solange sie die zentrale Funktion erfüllten, gemeinsam die Realität zu reparieren (Boltanski 2013). Als Metaphern fungieren die Alternativen Fakten dabei insofern, dass sie, um eine schöne Formulierung über die Anhänger_innen Donald Trumps zu paraphrasieren, von meinen Befragten offensichtlich „ernst, aber nicht beim Wort“ (Zito 2016) genommen werden. Dabei war augenfällig, dass untereinander eine Art stillschweigende Übereinkunft zu bestehen schien, die damit verbundenen Inkonsistenzen wegzulachen (und damit implizit anzuerkennen), sie aber nicht explizit zu thematisieren und gegebenenfalls nach außen trotzig zu leugnen. Sollte dies auch auf die von Feustel erwähnten Pegida-Demonstrant_innen zutreffen (was wir nur mutmaßen können), wäre dies auch eine plausible Erklärung dafür, warum sie auf das freundlich-zugewandte Interesse von Patzelt und seinen Mitarbeiter_innen mit Ablehnung reagieren: aus Angst, jemand könnte die gemeinsam genossene Realitätsfiktion, die von ihnen selbst nur halb angenommen wird, gefährden.

Auf dem Weg solcher Rekonstruktionen der sozial spezifischen lebensweltlichen Fundierung der Anschlussfähigkeit für rechte Mobilisierungen lassen sich Aussagen über die Affinität unterschiedlicher Träger_innengruppen gegenüber den Bewegungen der Rechten treffen. Solche Aussagen sind für politische Überlegungen zum strategischen Umgang mit der Mobilisierungsfähigkeit der Rechten fruchtbarer als zeitdiagnostische Großentwürfe wie der der ,Hyperrealität verschiedenen sozialen Träger_innengruppen identifizieren lassen, die auf verschiedene Gegenstrategien unterschiedlich ansprechen dürften: Denn selbst wenn alle Beteiligten bei Pegida, wie Feustel plausiblerweise vermutet, insgeheim wissen, dass die Befürchtung, man müsse Weihnachten bald in einer Moschee feiern, eher abwegig ist, macht es doch einen Unterschied, welche Funktion die Präsentation dieses alternativen Fakts für sie hat. Und die Vermutung liegt nahe, dass diese Funktion nicht für alle Beteiligten dieselbe ist. Solange wir aber nicht mit ihnen reden, werden wir das nie erfahren.

\section{Endnoten}

[1] Damit erledigen sich auch die gewichtigsten Einwände, die gemeinhin dagegen erhoben werden, im alltagssprachlichen Sinne mit Rechten zu reden. Weder bietet man ihnen eine Bühne noch normalisiert man ihre Position. Feustel reißt mit „Podien“ (Feustel 2019: 137) einen weiteren Problemkomplex an, der in dieser Entgegnung aber nicht wirklich diskutiert werden kann, nämlich den der Forschungskommunikation. Hier gelten die gerade genannten Einwände selbstverständlich weiter.

[2] Ohnehin ist das eine gute Faustregel für die qualitative Sozialforschung: Wenn die Interviewfrage mit der Forschungsfrage in eins fällt, sollten die Befragten das Buch selbst schreiben.

[3] Selbstverständlich ist dies nicht der einzig denkbare Weg der Beobachtung: Tagebücher, Propagandamaterialien, die zahllosen Youtube-Playlists mancher rechter Subkulturen und andere Quellen können gerade in Fällen, in denen die direkte Interaktion mit den Rechten nicht möglich ist oder nur unter unverhältnismäßigen Gefahren und Belastungen 
gesucht werden kann, genauso fruchtbare Gegenstände der Rekonstruktion sein. Allerdings ist das Interview oder die Gruppendiskussion, weil die Forschenden hier den unmittelbaren Interaktionskontext mitbeobachten und in damit in die Auswertung einbeziehen und gegebenenfalls die thematische Schwerpunktsetzung selbst bestimmen können, vermutlich der unkomplizierteste Weg.

\section{Autor innen}

Nils Kumkars Arbeitsschwerpunkte sind qualitative Methoden und soziologische Theorie, besonders die Erforschung von Lebensführung, Kritik und biographischer Enttäuschung. kumkar@uni-bremen.de

\section{Literatur}

Adorno, Theodor W. (1973): Studien zum autoritären Charakter. Frankfurt am Main: Suhrkamp.

Bohnsack, Ralf (2010): Rekonstruktive Sozialforschung. Opladen: Barbara Budrich.

Boltanski, Luc (2013): Rätsel und Komplotte. Kriminalliteratur, Paranoia, moderne Gesellschaft. Berlin: Suhrkamp.

Bourdieu, Pierre (1979): Entwurf einer Theorie der Praxis. Frankfurt am Main: Suhrkamp.

Bourdieu, Pierre (1993): Die öffentliche Meinung gibt es nicht. In: Pierre Bourdieu, Soziologische Fragen. Frankfurt am Main: Suhrkamp, 212-223.

Bourdieu, Pierre (2012). Was heißt Sprechen? Wien: New Academic Press.

Decker, Oliver / Kiess, Johannes / Brähler, Elmar (2015): Rechtsextremismus der Mitte und sekundärer Autoritarismus. Gießen: Psychosozial-Verlag.

Feustel, Robert (2019): Substanz und Supplement. Mit Rechten reden, zu Rechten forschen? Eine Einladung zum Widerspruch. In: $\mathrm{s} \mathrm{u} \mathrm{b} \backslash \mathrm{u} \mathrm{r}$ b a n. zeitschrift für kritische stadtforschung, $7(1 / 2), 137-145$.

Hochschild, Arlie (2017): Strangers in Their Own Land. Anger and Mourning on the American Right. New York: The New Press.

Horkheimer, Max (1937): Traditionelle und kritische Theorie. Zeitschrift für Sozialforschung 6/2, 245-294.

Kumkar, Nils Christian (2017): Autoritarismus und Realitätsverlust. Das Krisenerleben des klassischen Kleinbügertums und die Attraktivität Donald Trumps. Psychologie und Gesellschaftskritik 41 (3/4), 87-107.

Kumkar, Nils Christian (2018): The Tea Party, Occupy Wall Street, and the Great Recession. Basingstoke / New York: Palgrave Macmillan.

Oevermann, Ullrich (2005). Klinische Soziologie auf der Basis der Methodologie der objektiven Hermeneutik. Manifest der objektiv hermeneutischen Sozialforschung. http:// publikationen.ub.uni-frankfurt.de/volltexte/2005/540/ (letzter Zugriff am 16.2.2019).

Pollock, Friedrich (1955): Das Gruppenexperiment. Ein Studienbericht. Frankfurt am Main: Europäische Verlagsanstalt.

Rosenthal, Gabriele (1990): „Als der Krieg kam, hatte ich mit Hitler nichts mehr zu tun.“ Zur Gegenwärtigkeit des „Dritten Reiches“ in erzählten Lebensgeschichten. Opladen: Leske \& Budrich.

Zito, Salena (2016). Taking Trump Seriously, Not Literally. In: The Atlantic, 23.09.2016. https://www.theatlantic.com/politics/archive/2016/o9/trump-makes-his-case-in-pittsburgh/501335/ (letzter Zugriff am 16.2.2019). 
